\title{
Encouraging Persons with Hearing Problem to Learn Sign Language by Internet Websites
}

\author{
Mohammad Shirali-Shahreza \\ Computer Science Department \\ Sharif University of Technology \\ Tehran, IRAN \\ shirali@,cs.sharif.edu
}

\author{
Sajad Shirali-Shahreza \\ Computer Engineering Department \\ Sharif University of Technology \\ Tehran, IRAN \\ shirali@,ce.sharif.edu
}

\begin{abstract}
Nowadays the Internet users are from different ages and groups. Disabled people are a group of the Internet users. Some websites are especially created for these people. One group of the disabled people are deaf persons. They have a special talking language which is named sign language. Here we present a method to encourage them, esp. children, to learn the sign language. In this method, when a deaf person wants to enter a website which is created for deaf persons, a word is shown as a movie using a sign language. The user should recognize the word and select it from a list. If the user understands the sign language and recognizes the word, he/she can enter the website. This method is created based on a special HIP (Human Interactive Proofs) system which is designed for deaf persons. This project has been implemented by PHP scripting language.
\end{abstract}

Keywords: Deaf Persons, Disabled People, E-Learning, HIP (Human Interactive Proofs), Sign Language.

\section{Introduction}

Internet Expansion and development of the Internet in all aspects of daily life have been one of the great successes in recent years. One of the applications of the Internet is using it in learning and establishing e-learning systems.

Because of the growth of the Internet and the easy access to it, a great deal of private and personal information is available on the web.
On the other hands, nowadays the Internet in not only for special groups of people, but also people from any age and different groups are using the Internet. There are many websites for children on the Internet and the children using the Internet for various activities such as entertaining, educating, etc. The elderly people communicate and chat with their children and relative using the Internet.

One important group of Internet users is people with disability. Some of the Internet websites are especially developed for disabled persons. Some of these websites are designed for learning to disabled people.

One group of the disabled people are deaf persons. Deaf persons have a special talking language. They are using sign language which uses manual communication, body language and lip patterns instead of sound to convey meaning-simultaneously combining hand shapes, orientation and movement of the hands, arms or body, and facial expressions to express fluidly a speaker's thoughts.

Deaf persons should learn sign language as soon as possible to able to communicate with other peoples.

Here we present a method to encourage deaf persons, esp. children, to learn the sign language. For this purpose we use our invented method [1]. The used method is a kind of HIP methods.

HIP is abbreviation of Human Interactive Proofs and is applied to call systems in which human user proves their membership in a special group through a challenge/response protocol. Some examples are: human versus machine, user himself versus any other person, adult human versus a child, etc [2]. 
HIP methods are mostly used to tell human and machine apart and are called CAPTCHA (Completely Automatic Public Turing Test to Tell Computer and Human Apart). In the next section, we will discuss these methods in more details, but little work has been done in the field of telling users groups apart.

In our method, when a user wants to enter a website which is created for deaf persons, a word is shown to the user using sign language through a movie and he/she is asked to select the shown word from the list. In the third section, details of our method and its implementation will be mentioned.

In the final section, the conclusion will be made after investigating and studying some advantages of this method.

\section{CAPTCHA methods}

As mentioned before, most of the earlier projects in field of HIP is related to telling human and machine apart and referred to as CAPTCHA. In this section we study and examine CAPTCHA methods and its usages briefly.

CAPTCHA are systems which are used to distinguish between human and machine automatically. These systems are based on Artificial Intelligence (AI) topics. They are similar to Turing test [3], but they differ in that the judge is a computer [4]. The goal of these systems is to ask questions which human users can easily answer, while current computers cannot.

CAPTCHA methods can be generally divided into two groups: OCR-based and Non-OCR-Based.

In OCR-based methods, the image of a word is shown to user with distortion and various pictorial effects and he must type that word. Due to presence of various pictorial effects, the computer will encounter problems in the recognition of these words and only human user will be able to recognize the word. Examples of these methods include Gimpy [4] and Persian/Arabic Baffle Text [5]. But these methods usually result in dissatisfaction of users. On the other hand, efforts have been made for attacking these methods [6].

However, these methods usually disturb the users, therefore Non-OCR-based methods in which they do not use OCR systems have been presented so far. Examples of these methods include Collage CAPTCHA [7], PIX [8], and Text-to-Speech [9].

For example, in Collage CAPTCHA the images of some different objects (for example six objects such as airplane, car, apple, orange, pineapple and ball) are chosen. Then some effects such as rotating are done on the images and they are merged to create a single image. This image is shown to the user and he/she is asked to click on a certain image (for example on the image of the car) (Fig. 1).

The Non-OCR-Based methods are easier to work with than OCR-based methods and also can be used by disabled people.

The HIP method [1] which is used in this project is a Non-OCR-Based CAPTCHA method. This method will be described in the following section while explaining our proposed method.

\section{Our proposed method}

In this section, we explain our method which is designed for encouraging deaf persons to learn the sign language [1].

Our method is based on understanding the word which a person says using ASL (American Sign Language). In our method, a small movie is shown to the user. The movie shows a person which says a word using ASL. Then a list of words is shown to the user and he/she must choose the correct word. If the user chooses the correct answer, he/she passed the test and can continue using the website. Otherwise, a new test is generated for him/her.

Our method has been implemented by PHP programming language. In this implementation, at first a database of the movies of the words in sign language is collected. We used the movies which were created by Dr. David Stewart and his team at Michigan State University. These movies are available at the "ASL Browser" website [10]. There are more than 4500 words available in their library.

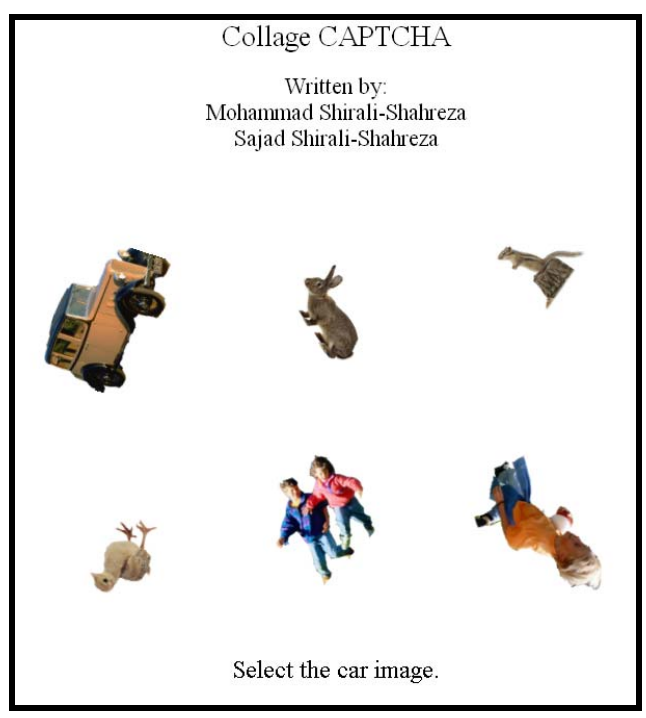

Figure 1. A sample of Collage CAPTCHA [7] 
For each word, the "ASL Browser" site has a movie shows a person saying the word using ASL (American Sign Language). The movies are in Quicktime (.MOV) format. Our database uses these movies and for each movie, we store the text of the word which the person says. The movie files have good quality and can be understood easily; while they have small sizes (average size of them is about $100 \mathrm{~KB}$ ).

To generate a test, the program chooses a random word from the database and shows the movie of that word to the user. Then it selects some other words (for example 3 other words). By showing more words, the difficulty of test is increased. Now the program shows all of these words to the user and asks him/her to select the word which is said in the movie.

When the user sends his/her answer, the program checks the answer and if the user chooses the right word, a message informs the user that he/she passed the test successfully and he/she will be allowed to enter the website. Otherwise, a message is shown to the user indicating that the answer was wrong. Then a new test is generated for him/her and shown to him/her.

A sample implementation of this project is available at: http://www.hip.ir/DeafHIP/ (Fig. 2).

In this site, 16 common words (such as "hello", "car" and "cat") are selected from the main database. The program chooses one of these words and shows the movie of this word to the user. Then the user is asked to select the shown word from a list of four words.

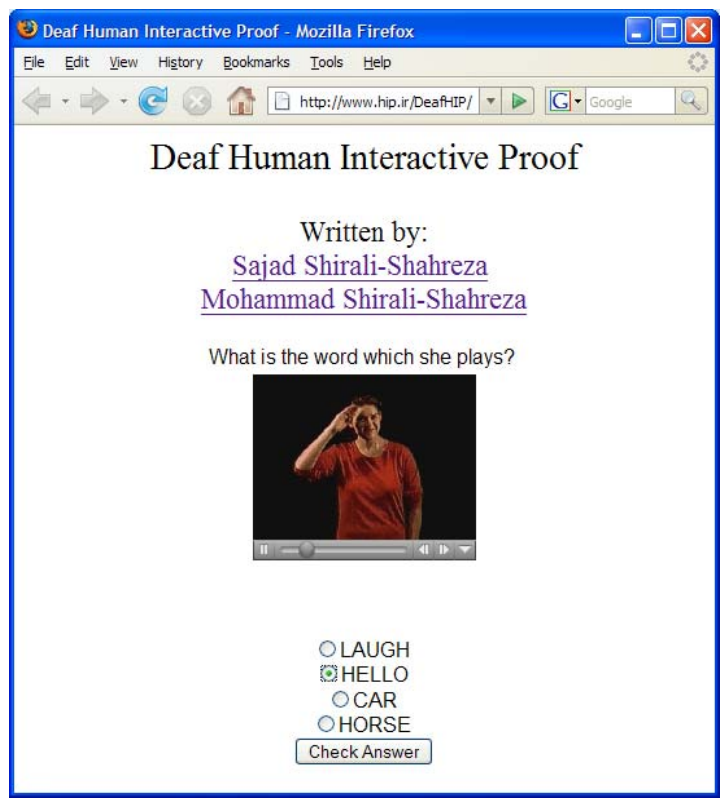

Figure 2. An screenshot of our project website [1]
Instead the above mentioned method, we can use another different method for asking a question from the user. The resources of this method are the same as above method. But in this method instead of asking the user to select the word which is shown by sign language, we select a word and show the movie of this word to the user along with the three movies of the other words. The user is asked select the select the movie of the asked word from a list of four shown movies.

While it seems that this method is easier for user to answer, but in this method the website loading time is high. However the download time can be reduced by lowing movie quality, convert it to grayscale, etc.

\section{Conclusion}

In this paper, a method is introduced for encouraging deaf persons, esp. children, to learn the sign language when using the Internet. In this method, a word is shown using sign language and the user should recognize the shown word and select its name from a list.

The difficulty of this method can be changed by changing the number of words which are shown to the user to select the correct answer from them.

This method only shows a small movie; therefore the website loading time is low. In addition, the download time can be reduced by lowing movie quality, convert it to grayscale, etc.

It does not require any processing on client side and can be used on small devices and devices with limited resources such as mobile phones. Also no keyboard is needed in this method because it only needs to select the word from a list. Therefore, it can be run on devices without a keyboard or devices in which it is difficult to use their keyboard; such as mobile phones.

This method is implemented entirely in PHP language. Because PHP is a well known open source and platform independent language, most of the web hosting companies support it, so this method can be easily integrated to available websites with low cost.

Instead of creating the database of sign language movies, we can use a text-to-sign language synthesis tool like [11] to create a movie for a word. By using this system, we don't need a large space for storing the movies. Also we can use similar tools to create cartoonish characters or convert the movies to cartoonish style (For example convert the movies to watercolor style) using special programs to attract the children.

Similar method can be created for other groups of disabled people. 


\section{References}

[1] S. Shirali-Shahreza and M. Shirali-Shahreza, "A New Human Interactive Proofs System for Deaf Persons," Proceedings of the $5^{\text {th }}$ International Conference on Information Technology: New Generations (ITNG 2008), Las Vegas, Nevada, USA, April 7-9, 2008.

[2] H.S. Baird and K. Popat, "Human Interactive Proofs and Document Image Analysis," Proceedings of the $5^{\text {th }} I A P R$ International Workshop on Document Analysis Systems, Springer LNCS 2423, pp. 507-518, 2002.

[3] A. Turing, "Computing Machinery and Intelligence", Mind, vol. 59, no. 236, pp. 433-460, October 1950.

[4] L. von Ahn, M. Blum, and J. Langford, "Telling Humans and Computers Apart Automatically," Communications of the ACM, vol. 47, no. 2, pp. 57-60, February 2004.

[5] M.H. Shirali-Shahreza and M. Shirali-Shahreza, "Persian/Arabic Baffletext CAPTCHA," Journal of Universal Computer Science (J.UCS), vol. 12, no. 12, pp. 1783-1796, December 2006.

[6] K. Chellapilla K. and P. Simard, "Using Machine Learning to Break Visual Human Interaction Proofs (HIPs),"
Advances in Neural Information Processing Systems (NIPS'2004), MIT Press, 2004.

[7] M. Shirali-Shahreza and S. Shirali-Shahreza, "Collage CAPTCHA," Proceedings of the $20^{\text {th }}$ IEEE International Symposium Signal Processing and Application (ISSPA 2007), 2007.

[8] M. Blum et al, The CAPTCHA Project (Completely Automatic Public Turing Test to tell Computers and Humans Apart), School of Computer Science, Carnegie-Mellon University. URL: http://www.captcha.net

[9] T.Y. Chan, "Using a text-to-speech synthesizer to generate a reverse Turing test," Proceedings of the $15^{\text {th }}$ IEEE International Conference on Tools with Artificial Intelligence (ICTAI 2003), pp. 226-232, 2003.

[10] D. Stewart et al., American Sign Language (ASL) Browser, Michigan State University, May 2007.

URL: http://commtechlab.msu.edu/sites/aslweb/

[11] M. Papadogiorgaki, N. Grammalidis, D. Tzovaras, and M.G. Strintzis: "Text-To-Sign Language Synthesis Tool," Proceedings of the $13^{\text {th }}$ European Signal Processing Conference, Antalya, Turkey, September 2005. 